\title{
A prospective, multicenter phase I/II study of induction chemotherapy with docetaxel, cisplatin and fluorouracil (DCF) followed by chemoradiotherapy in patients with unresectable locally advanced esophageal carcinoma
}

\author{
Hironaga Satake $^{1,2} \cdot$ Makoto Tahara $^{3} \cdot$ Satoshi Mochizuki $^{4} \cdot$ Ken Kato $^{5} \cdot$ Hiroki Hara $^{6}$. \\ Tomoya Yokota $^{7} \cdot$ Naomi Kiyota $^{8} \cdot$ Takayuki Kii $^{9}$ Keisho Chin ${ }^{10} \cdot$ Sadamoto Zenda $^{11}$. \\ Takashi Kojima $^{1} \cdot$ Hideaki Bando $^{1}$ - Tomoko Yamazaki ${ }^{1} \cdot$ Satoru Iwasa $^{5}$ • \\ Yoshitaka Honma $^{5}$ - Satoru Hamauchi ${ }^{7}$ - Takahiro Tsushima ${ }^{7} \cdot$ Atsushi Ohtsu $^{1}$
}

Received: 2 December 2015 / Accepted: 11 May 2016 / Published online: 18 May 2016 (C) The Author(s) 2016. This article is published with open access at Springerlink.com

\begin{abstract}
Purpose Standard care for unresectable locally advanced esophageal squamous cell carcinoma (ESCC) is concurrent chemoradiotherapy, but survival remains limited. Neoadjuvant chemotherapy with docetaxel, cisplatin and fluorouracil (DCF) has demonstrated promising activity, with a pathological complete response (CR) of $17 \%$ for resectable stage II/III ESCC. Here, we conducted a multicenter study to assess the efficacy and safety of induction chemotherapy with DCF followed by CRT in patients with unresectable locally advanced ESCC.

Methods Eligibility criteria included clinical T4 and/or M1 lymph node ESCC, PS 0-1 and age $20-70$ years. Treatment consisted of docetaxel $70 \mathrm{mg} / \mathrm{m}^{2}$ and cisplatin $70 \mathrm{mg} / \mathrm{m}^{2}$ on day 1 , and fluorouracil $750 \mathrm{mg} / \mathrm{m}^{2}$ on days $1-5$, repeated every 3 weeks for three cycles, followed by cisplatin
\end{abstract}

This article is an original report that was presented in part at the 49th American Society of Clinical Oncology Annual Meeting, Chicago, June 2, 2013.

Makoto Tahara

matahara@east.ncc.go.jp

1 Department of GI Oncology, National Cancer Center Hospital East, Kashiwa, Japan

2 Department of Medical Oncology, Kobe City Medical Center General Hospital, Kobe, Japan

3 Department of Head and Neck Medical Oncology, National Cancer Center Hospital East, 6-5-1, Kashiwanoha, Kashiwa 277-8577, Japan

4 Gastroenterology Division, Tokatsu-Tsujinaka Hospital, Abiko, Japan

5 Gastrointestinal Medical Oncology Division, National Cancer Center Hospital, Tokyo, Japan
$70 \mathrm{mg} / \mathrm{m}^{2}$ on days 64 and 92 , and fluorouracil $700 \mathrm{mg} / \mathrm{m}^{2}$ on days 64-67 and 92-95, concurrently with radiotherapy (60 Gy in 30 fractions, 5 days/week). Primary endpoint of the phase II part was CR rate.

Results Thirty-three patients were enrolled. The completion rate of protocol treatment was $88 \%$. Thirteen patients (39.4\%) achieved a CR. With a median follow-up period of 41 months (range 24-49 months), median progression-free survival was 12.2 months, and median survival was 26.0 months, with a survival rate of $40.4 \%$ at 3 years. The most common grade 3 or 4 toxicities were neutropenia, leukopenia, anorexia and dysphagia. No treatment-related death was observed.

Conclusion Induction chemotherapy with DCF followed by CRT is tolerable and shows promising efficacy for unresectable locally advanced ESCC.

Keywords Esophageal carcinoma $\cdot$ Squamous cell carcinoma $\cdot$ Induction chemotherapy $\cdot$ Chemoradiotherapy DCF

6 Department of Gastroenterology, Saitama Cancer Center, Saitama, Japan

7 Division of Gastrointestinal Oncology, Shizuoka Cancer Center, Shizuoka, Japan

8 Department of Oncology, Kobe University Hospital, Kobe, Japan

9 Department of Cancer Chemotherapy Center, Osaka Medical College, Osaka, Japan

10 Department of Gastroenterology, Cancer Institute Hospital of the Japanese Foundation for Cancer Research, Tokyo, Japan

11 Division of Radiation Oncology, National Cancer Center Hospital East, Kashiwa, Japan 


\section{Introduction}

Esophageal squamous cell carcinoma (ESCC) is a highly malignant disease. The high frequency of unresectable primary disease, distant metastases or medical unsuitability for surgery at initial diagnosis means that $40-60 \%$ of patients are not candidates for surgery, and their prognosis remains dismal [1]. Curative resection is not feasible in patients with locally advanced ESCC, particularly those with direct invasion of adjacent organs $\mathrm{T} 4$, and such cases have an unfavorable prognosis [2-4]. Standard care for unresectable locally advanced ESCC is concurrent chemoradiotherapy (CRT). A combination of cisplatin and fluorouracil $(\mathrm{CF})$ has become the standard regimen, but survival remains poor [5-7]. A previous study of CRT with CF plus 60 Gy of radiation in patients who had ESCC with T4 tumors and/or M1 lymph node metastasis (M1 LYM) showed a CR rate of 15-33\% and 3-year overall survival rate of $23-26 \%[3,8,9]$.

Docetaxel has shown activity against many solid tumors as monotherapy and in combination with other agents. Docetaxel has a different mechanism of action to $\mathrm{CF}$ and has been proved to have an additive effect with cisplatin and supra-additive antitumor activity with fluorouracil in vitro and in murine models of xenografted human tumors [10, 11]. Two phase III trials showed survival benefits from induction chemotherapy with docetaxel plus cisplatin and fluorouracil (DCF) compared to CF in locally advanced head and neck squamous cell carcinoma [12, 13]. Neoadjuvant chemotherapy with DCF demonstrated promising activity with a pathological CR rate of $17 \%$ for resectable stage II/III ESCC [14]. Results were also promising in a phase II trial of DCF followed by carboplatin and radiotherapy in locally advanced ESCC [15]. Furthermore, the risk of perforation of the esophageal wall related to definitive CRT has been highlighted in patients with T4 disease. To reduce the risk of perforation, we decided to use induction chemotherapy before CRT, with the aim of decreasing tumor volume before encountering severe esophagitis. However, the efficacy and safety of induction DCF followed by CF-RT for ESCC with T4 tumors and/or M1 lymph node metastasis (M1 LYM) has not been reported.

In this study, we aimed to assess the efficacy and safety of induction chemotherapy with DCF followed by CRT in patients with unresectable locally advanced ESCC.

\section{Materials and methods}

\section{Eligibility criteria}

Eligibility criteria were age 20-70 years; histologically proven squamous cell carcinoma of the esophagus; primary lesion located within the thoracic esophagus; no distant organ metastases; no esophagobronchial or esophagomediastinal fistulas; Eastern Cooperative Oncology Group (ECOG) performance status (PS) $<2$; adequate organ function defined by hemoglobin $\geq 10 \mathrm{~g} / \mathrm{dl}$, absolute neutrophil count $\geq 2 \times 10^{9} / 1$, platelet count $\geq 100 \times 10^{9} / 1$, total bilirubin $\leq 1.5 \mathrm{mg} / \mathrm{dl}$ and serum transaminases $\leq 3 \times$ the upper normal limit (UNL) of the institution and creatinine clearance $\geq 60 \mathrm{~mL} / \mathrm{min}, \mathrm{PaO}_{2} \geq 70 \mathrm{mmHg}$; and tumors judged unresectable by computed tomography (CT) scan defined by (1) primary tumor invasion depth $\mathrm{T} 4$ and/or (2) metastatic regional lymph node invasion to adjacent organs and/ or (3) M1 lymph node (M1 LYM). T4 was defined as a tumor that invades contiguous structures and M1 LYM as nodal metastasis beyond the regional lymph nodes, such as the supraclavicular or celiac lymph nodes (International Union Against Cancer TNM classification system, sixth edition). All areas of nodal disease had to be encompassable within the radiation field. Exclusion criteria were history of prior chemotherapy; myocardial infarction within the last 3 months; history of unstable angina pectoris, intestinal pneumonia, fibroid lung or severe emphysema; concurrent active malignancy; uncontrolled infection; and pregnancy or lactation. All patients were required to provide written informed consent before entering the study, which was approved by the institutional review board at each participating center.

\section{Study design and treatment}

The concurrent phase I part of the study was conducted in two cancer centers, and the subsequent phase II part was conducted in seven referral centers. Protocol treatment was defined as induction chemotherapy consisting of docetaxel (DTX), cisplatin (CDDP) plus fluorouracil (5-FU) (DCF) followed by chemoradiotherapy concurrent with CDDP plus 5-FU. Induction chemotherapy consisted of a 1-h intravenous (i.v.) administration of DTX on day 1; 2-h infusion of CDDP on day 1; and continuous i.v. administration of 5-FU on days $1-5$. This regimen was repeated every 3 weeks for a maximum of up to three cycles. Chemoradiotherapy consisted of concurrent administration of CDDP (70 $\mathrm{mg} / \mathrm{m}^{2}$ on days 64 and 92$)$ plus 5 -FU $\left(700 \mathrm{mg} / \mathrm{m}^{2}\right.$ on days 64-67 and 92-95) with radiotherapy.

Radiotherapy consisted of 60 Gy with a daily dose of 2 Gy and was delivered with $\geq 6$-MV X-rays. Three-dimensional treatment planning with a CT stimulator was used. Gross tumor volume (GTV) was determined by pretreatment with CT and GI endoscopy. Clinical target volume (CTV) included GTV with a craniocaudal margin of $3 \mathrm{~cm}$ in the primary site and a margin of $0-1 \mathrm{~cm}$ in lymph node metastases. Because target volume is always large in faradvanced esophageal cancer, no prophylactic irradiation 
for lymph node areas was performed. Completion of protocol treatment was defined as the end of induction DCF followed by concurrent CF-RT consisting of $60 \mathrm{~Gy}$ within 24 weeks from the date of first administration of induction DCF.

Prophylactic use of granulocyte colony-stimulating factor (G-CSF) was not allowed, but ciprofloxacin was administered on days $5-15$.

The phase I part was designed to determine the recommended dose (RD) of induction chemotherapy. Six patients were treated at dose level 1 (DTX $70 \mathrm{mg} / \mathrm{m}^{2}$, CDDP $70 \mathrm{mg} / \mathrm{m}^{2}$ and 5 -FU $750 \mathrm{mg} / \mathrm{m}^{2}$ ). If three or more of the six patients experienced a dose-limiting toxicity (DLT), six additional patients were accrued at the next lower dose level. The RD was defined as the dose at which two or fewer of six patients experienced a DLT. If two or fewer of the six patients in dose level 1 experienced a DLT, the RD was determined to be level 1 (DTX $70 \mathrm{mg} / \mathrm{m}^{2}$, CDDP $70 \mathrm{mg} / \mathrm{m}^{2}$ and $5-\mathrm{FU} 750 \mathrm{mg} / \mathrm{m}^{2}$ ).

The dose was modified for each patient based on hematologic or non-hematologic toxicity. DLT was defined as any of the following adverse events occurring within 28 days after completion of the protocol treatment: (1) febrile neutropenia lasting $>4$ days; (2) grade 4 thrombocytopenia $\left(<0.25 \times 10^{9} / 1\right)$; (3) grade 3 or 4 non-hematologic toxic effects, except grade 3 alopecia, anorexia, nausea, vomiting, constipation, stomatitis, esophagitis or infection due to stomatitis; (4) discontinuation of treatment due to an adverse event; or (5) treatment-related death.

In the subsequent phase II part, the enrolled patients were treated with the RD of induction chemotherapy followed by concurrent chemoradiotherapy as above.

\section{Study assessment}

Pretreatment evaluation included a medical history; physical examination; complete blood cell count and serum chemistry tests; esophagogastroduodenoscopy; and cervical, chest and abdominal CT scans. Endoscopic ultrasound, bronchoscopy and cervical ultrasound were optional. Adjacent organs were considered to be involved if the tumor extended into the lumen or caused a deformity of the airway in the trachea or tracheobronchial tree, and if the tumor was attached to the organ at a contact angle $\geq 90^{\circ}$ in the thoracic aorta as observed on the CT scan. T3 or lesser extent of disease was determined by endoscopic ultrasound. Lymph nodes were considered positive if they were $\geq 1 \mathrm{~cm}$ in diameter on any image. These evaluations for staging were reviewed, and cases were judged as potentially incurable with surgery by diagnostic radiologists as well as surgeons and medical oncologists at each institution.

All adverse events experienced during the study were recorded and graded according to the National Cancer
Institute Common Terminology Criteria for Adverse Events (CTCAE version 3.0). Late toxicity was graded according to the RTOG/EORTC Late Radiation Morbidity Scoring Scheme. Late toxicity was defined as toxicity occurring more than 31 days after treatment completion. Close follow-up using both endoscopy and CT was mandatory in the third week of every induction chemotherapy administration. If disease progression or new metastasis was detected, the subsequent cycle of induction chemotherapy was discontinued and a shift to chemoradiation was mandated. A history and physical examination, serum chemistry profile, cervical-chest-abdominal CT scan and esophagogastroduodenoscopy were performed in the fourth week after the completion of all protocol therapy. The following were performed every 3 months for 1 year and every 6 months thereafter until disease progression: physical examination, toxicity assessment, complete blood cell count, serum chemistry profile, cervical-chest-abdominal CT scan and esophagogastroduodenoscopy. Patterns of failure were defined as the first site of failure. Local/regional failure included the primary tumor and regional lymph nodes. Distant failure included any site beyond the primary tumor and regional lymph nodes.

\section{Endpoints and statistical analysis}

Once the RD of induction chemotherapy was determined in the first phase of the study, patient accrual continued for the phase II study, the main objective of which was to determine the objective response activity of this strategy. The primary endpoint was complete response (CR) rate evaluated according to the Response Evaluation Criteria in Solid Tumors (RECIST) criteria v1.0 and endoscopic assessment for the primary tumor. Primary tumor response was evaluated using endoscopy in accordance with the modified criteria of the tenth edition of Guidelines for the Diagnosis and Treatment of Carcinoma of the Esophagus, issued by the Japanese Society for Esophageal Diseases [16]. For the primary site, clinical CR was defined as disappearance of the primary tumor, ulceration and erosion as confirmed by endoscopic examination and negative biopsy results. A CR of lymph node metastasis was defined as the disappearance of all visible lymph node metastases on CT imaging. A CR was defined as a clinical CR of the primary tumor and $\mathrm{CR}$ of lymph node metastases. An evaluation of $\mathrm{CR}$ had to be confirmed by reassessment on endoscopy and CT 4 or more weeks later. Secondary endpoints included progression-free survival (PFS), overall survival (OS), response rate of induction chemotherapy, completion rate of protocol treatment and safety. We set the threshold objective CR rate at $30 \%$ and the expected objective CR rate at $50 \%$ on the basis of the results of previous studies [3,8]. Given a one-sided 
$\alpha$ of 0.1 and statistical power of $80 \%$, a minimum of 27 patients was required.

The survival curve was estimated using the KaplanMeier method. Safety and efficacy analyses were both conducted on an intention-to-treat (ITT) population, defined as all patients enrolled in the study who received at least one dose of induction chemotherapy. The PFS was defined as the time from the date of first administration of induction chemotherapy to the first documentation of disease progression, subsequent therapy or death. OS was determined from the date of first administration of induction chemotherapy to the date of death or last confirmation of survival. Statistical data were obtained using the SPSS software package (SPSS 22.0 Inc., Chicago, IL).

This trial was registered with University Hospital Medical Information Network (No. UMIN000003370).

\section{Results}

\section{Patient characteristics}

Thirty-three patients with histologically proven squamous cell carcinoma were enrolled from August 2009 to November 2011. The 33 patients are characterized in Table 1 . Baseline nutritional statuses were total protein median $7.0 \mathrm{~g} / \mathrm{dl}$ (range 5.9-8.0) and serum albumin level median $4.0 \mathrm{~g} / \mathrm{dl}$ (range 2.8-4.8). There were 16 patients (48\%) with T4 M0 disease, 13 (39\%) with non-T4 M1 LYM and 4 (12\%) with T4 M1 LYM. The site of clinical involvement in the 20 cases of T4 disease was the trachea in 15, trachea and thoracic aorta in 2 and thoracic aorta, pericardium and stomach in 1 case each.

Among the six patients who were registered to phase I, all patients were treated at the RD (level 1: DTX $70 \mathrm{mg} /$ $\mathrm{m}^{2}$, CDDP $70 \mathrm{mg} / \mathrm{m}^{2}$ and 5 -FU $750 \mathrm{mg} / \mathrm{m}^{2}$ ). Twenty-seven other patients were entered into phase II to further evaluate the tolerability and toxicity of the study regimen. All 33 patients were evaluated for toxicity and efficacy.

\section{Phase 1}

The first six patients were enrolled at dose level 1 (DTX $70 \mathrm{mg} / \mathrm{m}^{2}$, CDDP $70 \mathrm{mg} / \mathrm{m}^{2}$ and 5 -FU $750 \mathrm{mg} / \mathrm{m}^{2}$ ). No DLTs were observed, and hence, the RD was determined to be DTX $70 \mathrm{mg} / \mathrm{m}^{2}$, CDDP $70 \mathrm{mg} / \mathrm{m}^{2}$ and 5 -FU $750 \mathrm{mg} / \mathrm{m}^{2}$. All six patients completed the three courses of induction chemotherapy, and five patients received subsequent chemoradiation without cessation. The sixth patient required the temporary cessation of radiation therapy due to infection for 5 days, but completed all subsequent irradiation.
Table 1 Patient characteristics $(n=33)$

\begin{tabular}{lll}
\hline Variable & & $n(\%)$ \\
\hline Age & Median & 61 \\
& Range & $30-69$ \\
Sex & Male & $29(88)$ \\
ECOG PS & Female & $4(12)$ \\
& 0 & $19(58)$ \\
Tumor location & 1 & $14(42)$ \\
& Upper thorax & $18(55)$ \\
& Middle thorax & $13(39)$ \\
Clinical TNM and stage & Lower thorax & $2(6)$ \\
III & & \\
IVA & T4N1M0 & $16(48)$ \\
& T2N0M1a & $1(3)$ \\
& T2N1M1a & $1(3)$ \\
& T3N1M1a & 8 \\
IVB & T4N1M1a & 3 \\
& T3N1M1b & 3 \\
\hline
\end{tabular}

ECOG Eastern Cooperative Oncology Group, PS performance status

\section{Toxicity}

The worst toxicity throughout the treatment period is listed in Table 2. During the induction chemotherapy phase, grade 3 or 4 neutropenia and febrile neutropenia (FN) occurred in 72 and $6 \%$ of patients, respectively. Of these patients, 12 patients were administered G-CSF for FN or grade 4 neutropenia. One patient with grade $4 \mathrm{FN}$ developed pneumonia with shock, but recovered following treatment with G-CSF and antibiotics. Another patient with grade 4 FN, whose primary tumor involved the trachea at initial diagnosis, developed treatment-related perforation of the esophageal wall, leading to an esophagotracheal fistula after the first cycle of induction chemotherapy.

During the chemoradiation phase, grade 3 or 4 neutropenia and FN occurred in 18 and $3 \%$ of patients, respectively. Of these, one patient required G-CSF for 2 days starting from the 20th day of irradiation. One patient with T4 disease involving the trachea at initial diagnosis developed treatment-related perforation of the esophageal wall, and an esophagomediastinal fistula occurred on the 16th day from the start of irradiation. No treatment-related death was observed during the protocol treatment.

Six patients experienced late toxicities related to treatment, two with grade 2 esophageal stricture, two with grade 1 or 2 peripheral sensory neuropathy, one with grade 1 pleural effusion and one with grade 1 radiation pneumonitis. No patient experienced grade 3 or higher late toxicity. 
Table 2 Adverse events during the treatment period $(n=33)$

\begin{tabular}{|c|c|c|c|c|c|c|}
\hline & \multicolumn{6}{|c|}{ Grade } \\
\hline & 1 & 2 & 3 & 4 & All, \% & $3 / 4, \%$ \\
\hline \multicolumn{7}{|c|}{ Adverse events during induction chemotherapy } \\
\hline \multicolumn{7}{|c|}{ Hematologic } \\
\hline Leukopenia & 5 & 15 & 11 & 2 & 100 & 39 \\
\hline Neutropenia & 0 & 9 & 12 & 12 & 100 & 72 \\
\hline Anemia & 22 & 11 & 0 & 0 & 100 & 0 \\
\hline Thrombocytopenia & 22 & 11 & 0 & 0 & 100 & 0 \\
\hline \multicolumn{7}{|l|}{ Non-hematologic } \\
\hline Creatinine increased & 7 & 0 & 0 & 0 & 21 & 0 \\
\hline Elevation of AST & 14 & 0 & 0 & 0 & 42 & 0 \\
\hline Elevation of ALT & 10 & 9 & 1 & 0 & 30 & 3 \\
\hline Febrile neutropenia & - & - & 1 & 1 & 6 & 6 \\
\hline Hyperbilirubinemia & 3 & 1 & 0 & 0 & 12 & 0 \\
\hline Hyponatremia $(\geq \mathrm{G} 3)$ & - & - & 1 & 1 & 6 & 6 \\
\hline Alopecia & 14 & 6 & 0 & 0 & 60 & 0 \\
\hline Anorexia & 10 & 13 & 6 & 0 & 87 & 18 \\
\hline Constipation & 8 & 6 & 0 & 0 & 42 & 0 \\
\hline Diarrhea & 9 & 3 & 0 & 0 & 38 & 0 \\
\hline Dysphagia & 7 & 6 & 4 & 0 & 51 & 12 \\
\hline Edema & 2 & 0 & 1 & 0 & 9 & 3 \\
\hline Fever & 2 & 0 & 1 & 0 & 9 & 3 \\
\hline Fistula GI-esophagus & 0 & 0 & 1 & 0 & 3 & 3 \\
\hline Infection with normal ANC & 0 & 0 & 1 & 1 & 6 & 6 \\
\hline Nausea & 13 & 7 & 3 & 0 & 69 & 9 \\
\hline Rash & 3 & 0 & 0 & 0 & 9 & 0 \\
\hline Stomatitis & 11 & 6 & 1 & 0 & 54 & 3 \\
\hline Vomiting & 3 & 2 & 0 & 0 & 15 & 0 \\
\hline \multicolumn{7}{|c|}{ Adverse events during chemoradiation } \\
\hline \multicolumn{7}{|l|}{ Hematologic } \\
\hline Leukopenia & 5 & 15 & 8 & 0 & 85 & 24 \\
\hline Neutropenia & 11 & 10 & 6 & 0 & 82 & 18 \\
\hline Anemia & 6 & 22 & 1 & 0 & 88 & 3 \\
\hline Thrombocytopenia & 9 & 2 & 0 & 0 & 33 & 0 \\
\hline \multicolumn{7}{|l|}{ Non-hematologic } \\
\hline Creatinine increased & 8 & 1 & 0 & 0 & 27 & 0 \\
\hline Elevation of AST & 5 & 0 & 0 & 0 & 15 & 0 \\
\hline Elevation of ALT & 0 & 7 & 0 & 0 & 21 & 0 \\
\hline Febrile neutropenia & - & - & 1 & 0 & 3 & 3 \\
\hline Alopecia & 14 & 8 & 0 & 0 & 67 & 0 \\
\hline Anorexia & 12 & 6 & 4 & 0 & 67 & 12 \\
\hline Constipation & 9 & 0 & 0 & 0 & 27 & 0 \\
\hline Diarrhea & 4 & 0 & 0 & 0 & 12 & 0 \\
\hline Dysphagia & 8 & 8 & 5 & 0 & 64 & 15 \\
\hline Edema & 1 & 1 & 0 & 0 & 6 & 0 \\
\hline Esophagitis ( $\geq \mathrm{G} 3$ ) & - & - & 4 & 0 & - & 12 \\
\hline Fever & 2 & 0 & 0 & 0 & 6 & 0 \\
\hline Fistula GI-esophagus & 0 & 0 & 1 & 0 & 3 & 3 \\
\hline Infection with normal ANC & 1 & 0 & 1 & 0 & 6 & 3 \\
\hline Nausea & 12 & 3 & 3 & 0 & 55 & 9 \\
\hline
\end{tabular}


Table 2 continued

\begin{tabular}{lllllcc}
\hline & \multicolumn{2}{l}{ Grade } & & & \\
\cline { 2 - 6 } & 1 & 2 & 3 & 4 & All, \% & $3 / 4, \%$ \\
\hline Neuropathy sensory & 2 & 0 & 0 & 0 & 6 & 0 \\
Stomatitis & 4 & 0 & 0 & 0 & 12 & 0 \\
Vomiting & 3 & 1 & 0 & 0 & 12 & 0 \\
\hline
\end{tabular}

\section{Dose intensity}

During the induction chemotherapy phase, the median percentage of relative dose intensity (RDI) delivered was 83.6\% (range 33.0-100) for docetaxel, 81.5\% (range 33.0-100) for cisplatin and 84.1\% (range 33.2-100) for fluorouracil. The average RDI of induction chemotherapy was $83.0 \%$. Four of 33 patients $(12 \%)$ discontinued induction chemotherapy due to toxicity; of these, 3 discontinued after the first cycle of induction DCF, one each due to grade 4 hyponatremia, grade 4 febrile neutropenia and esophagotracheal fistula. One patient discontinued induction DCF after the second cycle due to grade 4 sepsis. During the chemoradiation phase, the median dose intensity delivery was $85.8 \%$ (range 30.0-100) for cisplatin and 94.1\% (range 49.9-100) for fluorouracil. The average RDI of chemotherapy was $90.0 \%$. Patients required dose reduction mainly due to bone marrow suppression, renal toxicity (creatinine clearance $<60 \mathrm{~mL}$ / min) or stomatitis. The completion rate of protocol treatment was $87.8 \%$.

\section{Response to treatment}

Response to induction chemotherapy and chemoradiotherapy is summarized in Table 3. Two of the 33 patients had CR after induction chemotherapy, and thirteen patients $(39.4 \%, 95 \%$ CI 21.8-57.0 \%) had CR after chemoradiation.

\section{Survival and patterns of failure}

With a median follow-up period of 41 months (range 24-49 months), median survival was 26.0 months (95\% CI 11.8-40.2), and 1- and 3-year survival rates were $78.8 \%$ (95 \% CI 60.6-89.3) and 40.4\% (95\% CI 23.356.9), respectively (Fig. 1). The median time to progression was 12.2 months (95\% CI 8.2-16.2), and 1- and 3-year progression-free survival rates were $51.5 \%(95 \%$ CI 33.5-66.9) and 27.3\% (95\% CI 13.6-42.9), respectively (Fig. 2). No significant difference ( $p=0.911)$ was seen in survival benefit between T4 and non-T4 groups, with an overall survival time of 26.0 versus 25.6 months, respectively.
Table 3 Response rate to treatment $(n=33)$

\begin{tabular}{lcc}
\hline Variable & $n$ & $\%(95 \% \mathrm{CI})$ \\
\hline Response after induction chemotherapy & \\
Overall & 20 & $60.6(43.7-77.5)$ \\
Complete response & 2 & $6.1(0-14.7)$ \\
Partial response & 18 & $54.5(36.6-72.5)$ \\
Response after CRT & & \\
Overall & 24 & $72.7(55.8-84.9)$ \\
Complete response & 13 & $39.4(24.7-56.3)$ \\
Partial response & 11 & $33.3(19.8-50.4)$ \\
\hline
\end{tabular}

$C I$ confidence interval, $C R T$ chemoradiation

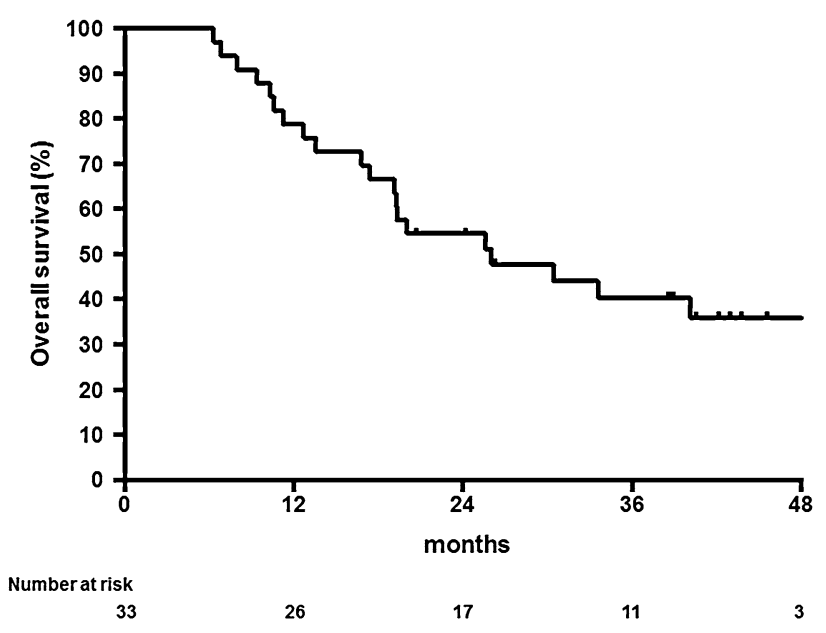

Fig. 1 Kaplan-Meier estimates of overall survival $(n=33)$

Among all 33 patients, 21 (64\%) received post-protocol treatment. Eight patients underwent salvage surgery, with five cases of curative resection. Three patients underwent salvage endoscopic resection, with two cases of pathological complete resection. Three cases went off-protocol due to adverse events during induction chemotherapy and received definitive chemoradiotherapy (two with CF-RT and one with 5-FU/nedaplatin-RT), with one case of complete response. One patient received definitive chemoradiotherapy with a $\mathrm{CF}$ regimen due to recurrence out of a prior radiation field. Nine patients received palliative chemotherapy for progression. 


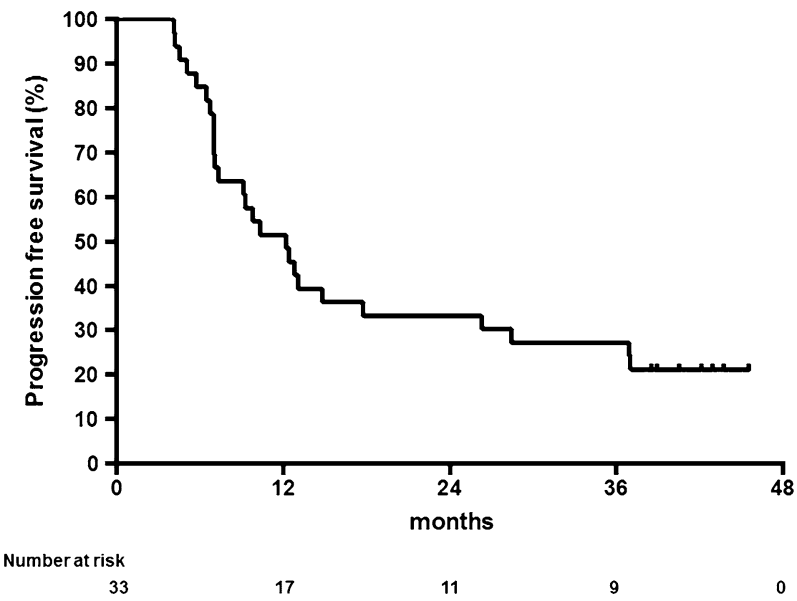

Fig. 2 Kaplan-Meier estimates of progression-free survival $(n=33)$

Table 4 Patterns of failure $(n=33)$

\begin{tabular}{lrr}
\hline & $n$ & $\%$ \\
\hline Alive/no failure & 8 & 24 \\
Any failure & 25 & 76 \\
Persistent local disease & 18 & 55 \\
Local recurrence & 1 & 3 \\
Regional lymph node recurrence & 3 & 9 \\
Distant recurrence & 3 & 9 \\
\hline
\end{tabular}

Patterns of first failure are shown in Table 4. All failures occurred within 1 year (range 6.1-8.0 months) after confirmation of clinical CR.

\section{Discussion}

In this multicenter prospective trial of induction chemotherapy followed by concurrent CRT, induction DCF showed substantial activity in patients with unresectable locally advanced ESCC. Previous studies of concurrent chemoradiotherapy with cisplatin and 5-FU in patients with ESCC with T4 tumors and/or M1 LYM showed a clinical complete response rate of 15-33\% with a median PFS of 6 months and a 3-year overall survival rate of 23-26\% [3, $8,9]$. Our present study did not achieve the expected CR rate $(39.4 \%)$, but did demonstrate promising efficacy, with a median PFS of 12 months and a 3-year survival rate of $40.4 \%$.

The reason for not achieving the expected $\mathrm{CR}$ rate is unclear. The recent randomized phase 3 trials with induction chemotherapy for locally advanced head and neck squamous cell carcinoma showed survival benefits for DCF compared to $\mathrm{CF}$, but the percentages of patients with a complete response and overall response did not statistically differ [12]. Patterns of failure in our present study showed that the distant recurrence rate was quite low $(9 \%)$. These might mean that this induction chemotherapy contributes to survival benefit by controlling minor metastases, but does not contribute to clinical response. The prolongation of PFS was clearly aided by the induction chemotherapy. Furthermore, post-protocol treatment, such as salvage surgery/endoscopic resection and palliative chemotherapy, might also have contributed to the regimen's survival benefit. Only esophagectomy has curative potential, but salvage surgery is associated with high morbidity rates [17-19]. The participating centers in our present study are regarded as highly specialized, and complications of salvage surgery might therefore have been minimized. Salvage endoscopic resection has curative intent for patients with locoregional failure after definitive CRT for ESCC [20]. Moreover, salvage chemotherapy with taxanes has shown a clinical benefit for ESCC in patients who previously received platinum-based chemotherapy [21, 22]. While Ohtsu et al. [8] reported that survival rate with $\mathrm{T} 4$ disease was inferior to that with non-T4 disease, we saw no significant difference in response rate or survival benefit between our T4 and non-T4 groups, with an overall survival time of 26.0 versus 25.6 months, respectively.

Of the 13 patients who had no response after induction chemotherapy (clinical SD or PD), 2 achieved clinical CR and 4 achieved clinical PR after subsequent definitive CRT. A shift in strategy to definitive CRT might therefore be effective for non-responders to induction chemotherapy.

Although induction DCF induced severe neutropenia, as expected, febrile neutropenia occurred in only $6 \%$ due to prophylactic use of ciprofloxacin. The risk of perforation of the esophageal wall related to definitive CRT has been highlighted in patients with T4 disease. Previous studies reported a frequency of perforation of the esophageal wall of 14-23\% in patients with T4 disease who received CRT $[8,9]$. To reduce the risk of perforation, we decided to use induction chemotherapy before CRT, with the aim of decreasing tumor volume before encountering severe esophagitis. Treatment-related perforation of the esophageal wall occurred in only one patient during induction chemotherapy ( $3 \%$ ) and in a second patient during CRT (3\%). This incidence is lower than in the previous study [8, 9], suggesting that this strategy was effective, albeit with room for improvement. We did not mandate bronchoscopy to evaluate the degree of invasion of the trachea before treatment; doing so in T4 tracheal lesions before treatment will allow the prediction of perforation risk.

Induction with DCF followed by concurrent CRT using carboplatin was previously reported to have a CR rate of 
$16 \%$ and median overall survival of 10.8 months [15]. Further, Higuchi et al. [23] reported that definitive CRT with DCF (DCF-R) for locally advanced ESCC had a high clinical CR rate $(52.4 \%)$ as well as prolonged PFS (median 11.1 months) and OS (MST 29.0 months). Although these survival data were equivalent to those of our present study, DCF-R was associated with a relatively high incidence of FN (grade 3 or more, $38.1 \%$ ) and late toxic effects, namely grade 3 or more pericardial effusion $(2.6 \%)$, esophagusrelated toxicities $(7.7 \%)$ and cardiovascular toxicities $(2.6 \%)$. Accordingly, the use of induction chemotherapy followed by concurrent CRT or definitive DCF-R using three cytotoxic drugs for T4 and/or M1 LYM is still under discussion.

Several limitations of the study warrant mention. The total dose of irradiation for locally advanced ESCC is still not standardized. The Intergroup 0123 study found no improvement in survival or locoregional control when comparing a radiotherapy dose of 64.8 Gy with 50.4 Gy [24]. On this basis, the standard radiation dose for definitive CRT is now 50.0-50.4 Gy in the USA. In Japan, definitive chemoradiotherapy with $\geq 60$ Gy has been employed to treat locally advanced ESCC, especially for T4 and/or M1 LYM [3, 8, 25]. Irradiation in our present study consisted of 60 Gy with a daily dose of 2 Gy. However, highdose irradiation might increase the risk of perforation of the esophageal wall in the chemoradiation phase. Although the study included the efficacy and safety of induction DCF followed by CRT for patients with locally unresectable ESCC, it was conducted at expert centers in Japan. Thus, a conclusive answer to the optimum strategy for locally unresectable ESCC will require prospective randomized controlled trials at multiple institutions with a larger number of patients.

In conclusion, this study showed that induction chemotherapy with DCF followed by CRT was tolerable, and might feasible in patients with unresectable locally advanced ESCC.

\begin{abstract}
Acknowledgments The authors would like to thank the patients and families who participated in this study. We also thank Shigenori Kadowaki and Fumio Nagashima for their membership of the independent review committee for safety and efficacy, as well as Tomoko Ichimura and Eri Horiguchi for technical support.
\end{abstract}

\section{Compliance with ethical standards}

Conflict of interest Makoto Tahara, MD, Ph.D., has received grant/ research support from Eisai, and Merck Sharp \& Dohme, and has received honoraria from Merck Serono, Bristol-Myers Squibb, Eisai, Otsuka and Bayer. Tomoya Yokota, MD, Ph.D., serves in an advisory role to AstraZeneca and Merck Serono and has received lecture fees from Merck Serono, Bristol-Myers Squibb, Takeda Pharmaceutical Co., Ltd., Niphix and Otsuka Pharmaceutical Co., Ltd. Naomi Kiyota, MD, Ph.D., has received honoraria from Bristol-Myers Squibb. All remaining authors have declared no conflicts of interest.
Open Access This article is distributed under the terms of the Creative Commons Attribution 4.0 International License (http://creativecommons.org/licenses/by/4.0/), which permits unrestricted use, distribution, and reproduction in any medium, provided you give appropriate credit to the original author(s) and the source, provide a link to the Creative Commons license, and indicate if changes were made.

\section{References}

1. Sagar PM, Gauperaa T, Sue-Ling H, McMahon MJ, Johnston D (1994) An audit of the treatment of cancer of the oesophagus. Gut 35(7):941-945

2. Ikeda K, Ishida K, Sato N, Koeda K, Aoki K, Kimura Y, Iwaya T, Ogasawara S, Iijima S, Nakamura R, Uesugi N, Maesawa C, Saito K (2001) Chemoradiotherapy followed by surgery for thoracic esophageal cancer potentially or actually involving adjacent organs. Dis Esophagus 14(3-4):197-201

3. Ishida K, Ando N, Yamamoto S, Ide H, Shinoda M (2004) Phase II study of cisplatin and 5-fluorouracil with concurrent radiotherapy in advanced squamous cell carcinoma of the esophagus: a Japan Esophageal Oncology Group (JEOG)/Japan Clinical Oncology Group trial (JCOG9516). Jpn J Clin Oncol 34(10):615-619. doi:10.1093/jjco/hyh107

4. Matsubara T, Ueda M, Kokudo N, Takahashi T, Muto T, Yanagisawa A (2001) Role of esophagectomy in treatment of esophageal carcinoma with clinical evidence of adjacent organ invasion. World J Surg 25(3):279-284. doi:10.1007/s002680020060

5. Bedenne L, Michel P, Bouche O, Milan C, Mariette C, Conroy T, Pezet D, Roullet B, Seitz JF, Herr JP, Paillot B, Arveux P, Bonnetain F, Binquet C (2007) Chemoradiation followed by surgery compared with chemoradiation alone in squamous cancer of the esophagus: FFCD 9102. J Clin Oncol 25(10):1160-1168. doi:10.1200/JCO.2005.04.7118

6. Herskovic A, Martz K, al-Sarraf M, Leichman L, Brindle J, Vaitkevicius V, Cooper J, Byhardt R, Davis L, Emami B (1992) Combined chemotherapy and radiotherapy compared with radiotherapy alone in patients with cancer of the esophagus. N Engl J Med 326(24):1593-1598. doi:10.1056/NEJM199206113262403

7. Cooper JS, Guo MD, Herskovic A, Macdonald JS, Martenson JA Jr, Al-Sarraf M, Byhardt R, Russell AH, Beitler JJ, Spencer S, Asbell SO, Graham MV, Leichman LL (1999) Chemoradiotherapy of locally advanced esophageal cancer: long-term followup of a prospective randomized trial (RTOG 85-01). Radiation Therapy Oncology Group. JAMA 281(17):1623-1627

8. Ohtsu A, Boku N, Muro K, Chin K, Muto M, Yoshida S, Satake M, Ishikura S, Ogino T, Miyata Y, Seki S, Kaneko K, Nakamura A (1999) Definitive chemoradiotherapy for T4 and/or M1 lymph node squamous cell carcinoma of the esophagus. J Clin Oncol 17(9):2915-2921

9. Shinoda M, Ando N, Kato K, Ishikura S, Kato H, Tsubosa Y, Minashi K, Okabe H, Kimura Y, Kawano T, Kosugi S, Toh Y, Nakamura K, Fukuda H, Japan Clinical Oncology G (2015) Randomized study of low-dose versus standard-dose chemoradiotherapy for unresectable esophageal squamous cell carcinoma (JCOG0303). Cancer Sci 106(4):407-412. doi:10.1111/ cas. 12622

10. Catimel G, Verweij J, Mattijssen V, Hanauske A, Piccart M, Wanders J, Franklin H, Le BN, Clavel M, Kaye SB (1994) Docetaxel (Taxotere): an active drug for the treatment of patients with advanced squamous cell carcinoma of the head and neck. EORTC Early Clinical Trials Group. Ann Oncol 5(6):533-537

11. Dreyfuss AI, Clark JR, Norris CM, Rossi RM, Lucarini JW, Busse PM, Poulin MD, Thornhill L, Costello R, Posner MR 
(1996) Docetaxel: an active drug for squamous cell carcinoma of the head and neck. J Clin Oncol 14(5):1672-1678

12. Posner MR, Hershock DM, Blajman CR, Mickiewicz E, Winquist E, Gorbounova V, Tjulandin S, Shin DM, Cullen K, Ervin TJ, Murphy BA, Raez LE, Cohen RB, Spaulding M, Tishler RB, Roth B, Viroglio Rdel C, Venkatesan V, Romanov I, Agarwala S, Harter KW, Dugan M, Cmelak A, Markoe AM, Read PW, Steinbrenner L, Colevas AD, Norris CM Jr, Haddad RI (2007) Cisplatin and fluorouracil alone or with docetaxel in head and neck cancer. N Engl J Med 357(17):1705-1715. doi:10.1056/ NEJMoa070956

13. Vermorken JB, Remenar E, van Herpen C, Gorlia T, Mesia R, Degardin M, Stewart JS, Jelic S, Betka J, Preiss JH, van den Weyngaert D, Awada A, Cupissol D, Kienzer HR, Rey A, Desaunois I, Bernier J, Lefebvre JL (2007) Cisplatin, fluorouracil, and docetaxel in unresectable head and neck cancer. N Engl J Med 357(17):1695-1704. doi:10.1056/NEJMoa071028

14. Hara H, Tahara M, Daiko H, Kato K, Igaki H, Kadowaki S, Tanaka Y, Hamamoto Y, Matsushita H, Nagase M, Hosoya Y (2013) Phase II feasibility study of preoperative chemotherapy with docetaxel, cisplatin, and fluorouracil for esophageal squamous cell carcinoma. Cancer Sci 104(11):1455-1460. doi:10.1111/ cas. 12274

15. Chiarion-Sileni V, Corti L, Ruol A, Innocente R, Boso C, Del Bianco P, Pigozzo J, Mazzarotto R, Tomassi O, Ancona E (2007) Phase II trial of docetaxel, cisplatin and fluorouracil followed by carboplatin and radiotherapy in locally advanced oesophageal cancer. Br J Cancer 96(3):432-438. doi:10.1038/sj.bjc.6603585

16. Japanese classification of esophageal cancer, 10th ed (2008). Parts II and III. Esophagus 6(2):71-94

17. Nakamura T, Hayashi K, Ota M, Eguchi R, Ide H, Takasaki K, Mitsuhashi N (2004) Salvage esophagectomy after definitive chemotherapy and radiotherapy for advanced esophageal cancer. Am J Surg 188(3):261-266. doi:10.1016/j.amjsurg.2004.06.003

18. Tomimaru Y, Yano M, Takachi K, Miyashiro I, Ishihara R, Nishiyama K, Sasaki Y, Ishikawa O, Doki Y, Imaoka S (2006) Factors affecting the prognosis of patients with esophageal cancer undergoing salvage surgery after definitive chemoradiotherapy. J Surg Oncol 93(5):422-428. doi:10.1002/jso.20475
19. Swisher SG, Wynn P, Putnam JB, Mosheim MB, Correa AM, Komaki RR, Ajani JA, Smythe WR, Vaporciyan AA, Roth JA, Walsh GL (2002) Salvage esophagectomy for recurrent tumors after definitive chemotherapy and radiotherapy. J Thorac Cardiovasc Surg 123(1): 175-183

20. Yano T, Muto M, Hattori S, Minashi K, Onozawa M, Nihei K, Ishikura S, Ohtsu A, Yoshida S (2008) Long-term results of salvage endoscopic mucosal resection in patients with local failure after definitive chemoradiotherapy for esophageal squamous cell carcinoma. Endoscopy 40(9):717-721. doi:10.105 5/s-2008-1077480

21. Kato K, Tahara M, Hironaka S, Muro K, Takiuchi H, Hamamoto Y, Imamoto H, Amano N, Seriu T (2011) A phase II study of paclitaxel by weekly 1-h infusion for advanced or recurrent esophageal cancer in patients who had previously received platinum-based chemotherapy. Cancer Chemother Pharmacol 67(6):1265-1272. doi:10.1007/s00280-010-1422-x

22. Muro K (2004) A phase II study of single-agent docetaxel in patients with metastatic esophageal cancer. Ann Oncol 15(6):955-959. doi:10.1093/annonc/mdh231

23. Higuchi K, Komori S, Tanabe S, Katada C, Azuma M, Ishiyama H, Sasaki T, Ishido K, Katada N, Hayakawa K, Koizumi W, Kitasato Digestive D, Oncology G (2014) Definitive chemoradiation therapy with docetaxel, cisplatin, and 5-fluorouracil (DCF-R) in advanced esophageal cancer: a phase II trial (KDOG 0501P2). Int J Radiat Oncol Biol Phys 89(4):872-879. doi:10.1016/j. ijrobp.2014.03.030

24. Minsky BD, Pajak TF, Ginsberg RJ, Pisansky TM, Martenson J, Komaki R, Okawara G, Rosenthal SA, Kelsen DP (2002) INT 0123 (Radiation Therapy Oncology Group 94-05) phase III trial of combined-modality therapy for esophageal cancer: high-dose versus standard-dose radiation therapy. J Clin Oncol 20(5):1167-1174

25. Ohtsu A, Yoshida S, Boku N, Fujii T, Miyata Y, Hosokawa K, Koba I, Shimizu W, Ogino T (1995) Concurrent chemotherapy and radiation therapy for locally advanced carcinoma of the esophagus. Jpn J Clin Oncol 25(6):261-266 